\title{
Erratum to: Unusual \\ Diseases with Common Symptoms
}

\author{
Anthony M. Szema
}

\section{Erratum to:}

A.M. Szema (ed.), Unusual Diseases with Common Symptoms, https://doi.org/10.1007/978-3-319-58952-7

The following corrections were missed out during the proofs corrections stage and are now amended.

- On page vii (Preface), the word "and" was removed and the word "Sincerely" was moved to the end of the preface before the editor's name.

- On page 13 (Chapter 2), the word "Diazepam" was changed to "diazepam".

- On page 139 (Chapter 14), the text "Photo courtesy of Charles Marboe, MD, Columbia University Medical Center" was removed from the caption of Fig. 14.2.

- On page 147 (Chapter 15), the text "a type of" was removed from the last paragraph.

- On page 205 (Chapter 21), "NYU" was changed to "New York University" in the author affiliation.

The updated online version of this book can be found at https://doi.org/10.1007/978-3-319-58952-7

A. M. Szema $(\square)$

Columbia University Child and Adolescent Psychiatric

Epidemiology Group CDC NIOSH U01 OH011308 “9/11 Trauma and Toxicity in Childhood: Longitudinal Health and Behavioral Outcomes", New York, USA 